\title{
Observations on the Diagnostic Effects of CT Examination (Enhanced Scan) on Hemorrhage Corpus Luteum Cyst
}

\author{
Xiang Gao, Heng Tang*, Lianglong Wu, Zou Mei, Liangjin Liu, Junying Bi \\ Hubei No.3 People's Hospital of Jianghan University, Wuhan 430033, Hubei Province, China
}

\begin{abstract}
Objective: To investigate the diagnostic effects of enhanced CT scan on hemorrhage corpus luteum cyst (HCLC). Methods: The clinical data of 24 patients with hemorrhage corpus luteum cyst diagnosed by surgery and pathology in our hospital were collected. All patients received enhanced CT scan to evaluate the diagnostic value of CT. Results: The average diameter of the cysts in this group of patients was $5.1 \mathrm{~cm}$ by CT. Further detection of the ruptured cyst wall showed that there was a liquid lowdensity shadow. Some of the cysts were accompanied by signs of increased density, indicating the presence of blood clots; all patients received surgical treatment. Intraoperative ultrasound examination of the cysts had an average diameter of $5.2 \mathrm{~cm}$ and a wall of $0.2 \sim 0.4 \mathrm{~mm}$. Among them, 19 cases of cysts contained "coffee-colored" liquid with varying degrees of blood clots, and 5 cases had no blood clots in the cysts but there were a lot of blood clots beside the cysts. Conclusion: The application of enhanced CT scan in patients with hemorrhage corpus luteum cyst is of great value. It can provide accurate data reference for clinical treatment and is worthy of promoting its clinical application.
\end{abstract}

Keywords: Enhanced CT scan; Hemorrhage corpus luteum cyst; Diagnostic effects

Publication date: November, 2020

Publication online: 30 November, 2020

"Corresponding author: Heng Tang, 358282436@ qq.com

Hemorrhage corpus luteum cyst (HCLC) is a gynecological acute abdomen, which is more common among women of childbearing age. It has the characteristics of rapid onset and rapid progress.
Patients mainly suffer from manifestations such as sudden lower abdominal pain, peritoneal irritation, and intrapelvic hemorrhage, etc. Clinically, it is often misdiagnosed as ectopic pregnancy, ovarian cyst pedicle torsion or acute appendicitis. If the diagnosis is not timely, hemorrhagic shock will occur, which will threaten the patient's life. The treatment of HCLC emphasizes targeted and individualized treatment, that is, those with less bleeding can receive conservative treatment to avoid surgical trauma; while for those with more bleeding, surgical treatment should be carried out in time to improve the survival rate of patients ${ }^{[1]}$. Regarding this, strengthening the assessment of patients' early bleeding is of great significance. Existing studies believe that the use of enhanced CT scan to diagnose HCLC is of great value. In view of this opinion, this paper collected and analyzed the clinical data of 24 patients with hemorrhage corpus luteum cyst diagnosed by surgery and pathology in our hospital. The report is as follows.

\section{Information and Methods}

\subsection{General Information}

Taking the period from March 2014 to June 2018 as the research period, the clinical data of 24 patients with hemorrhage corpus luteum cyst diagnosed by surgery and pathology in our hospital during this period were collected. The patients in this group aged 18 to 38 years old, with an average age of $(25.16 \pm 2.16)$ years old, of which 19 cases had a reproductive history and 5 cases had no reproductive history, all had a history of sexual intercourse. All patients admitted to the hospital with severe abdominal pain as the first symptom. 
Among them, 16 cases were accompanied by nausea and vomiting, 12 cases were accompanied by anal bulging sensation, and 1 case had a small amount of vaginal bleeding. 12 cases of patients started having symptoms after commencing sexual life. There were 6 cases of onset after exercise, 4 cases of onset after stool, and 2 cases of no significant cause. Before receiving CT examination, they all received ultrasound examination, which showed that there was a large amount of abdominal and pelvic fluid.

\subsection{Methods}

Model: Philips Brilliance CT or GE Revolution CT scanner was chosen, with a slice thickness of $1.5 \mathrm{~mm}$ and a slice distance of $1.5 \mathrm{~mm}$. All patients in this group underwent enhanced CT scan. $80-100 \mathrm{ml}$ contrast agent was injected before the scan. The delay time of the arterial phase was $28 \mathrm{~s}$, and the delay time of the venous phase was 60 s. After obtaining the original scan parameters, they were transferred to the workstation for post-processing. All acquired images were analyzed by senior imaging physicians in the undergraduate room. For those with atypical CT signs, joint analyses by $\geq 3$ physicians were conducted to obtain a unified opinion. For differences in diagnosis, the first diagnosis and the second diagnosis were made.

\section{Results}

Under enhanced CT scanning, the lesions of 24 patients in this group were found to be more common on the right side. Among them, 9 patients also had luteal cysts on the left ovaries, which were not ruptured, as shown in Table 1. The average diameter of cysts in this group was $5.1 \mathrm{~cm}$ by CT. The ruptured cyst wall detected liquid low-density shadow in the ventricle. Some of the cysts were accompanied by signs of increased density, indicating the presence of blood clots. All patients received surgical treatment. The average diameter of the cysts was $5.2 \mathrm{~cm}$ and the cyst wall was $0.2 \sim 0.4 \mathrm{~mm}$ during the operation. Among them, 19 cases of cysts contained "coffeecolored" liquid with varying degrees of clots. In 5 cases, there were no blood clots in the cysts but a large number of blood clots were found beside the cysts.

Table 1. Location of Cyst Rupture in this Group of Patients ( $n, \%)$

\begin{tabular}{ccc}
\hline Site of HCLC by CT & No. of Cases & Percentage \\
\hline Left Side & 9 & $37.5 \%$ \\
Right Side & 15 & $62.5 \%$ \\
Both Sides & 0 & $0.00 \%$ \\
\hline
\end{tabular}

\section{Discussions}

Ovarian corpus luteum is mostly formed after ovulation and is affected by luteinizing hormones. After ovulation, mature follicles, granulosa cells and follicular membrane cells, etc. will undergo a large number of proliferation and hypertrophy, thereby forming ovarian corpus luteum. After eliminating unfertilized and implanted egg, the corpus luteum usually shrinks after 10 days after ovulation under normal circumstances, and the life span is about 14 days. If the corpus luteum is present in the patient's abdominal cavity for a long time, and its effusion gradually increases, and the volume gradually increases over $3 \sim 10 \mathrm{~cm}$, this pathological change is judged as a corpus luteum cyst. As the lesion is large in size and mostly appears on the surface of the ovary, it lacks of elasticity and is fragile, highly tense, and rich in blood vessels. If affected by external forces, such as sex life, gynecological examination and exercise, etc., it may result in cyst rupture and bleeding ${ }^{[2]}$. Previous studies have confirmed that the corpus luteum cyst of the ovary continuously secretes hormones, leading to delayed menstrual cycle. Therefore, HCLC is more common after the menstrual cycle, and the cause of misdiagnosis is mainly related to early pregnancy and menopause. Therefore, misdiagnosis of ectopic pregnancy may occur in clinical diagnosis.

Ovarian corpus luteum cyst rupture is more common in the second half of the menstrual cycle. Ovarian corpus luteum cyst rupture includes spontaneous rupture and external force induced rupture. The reasons for the ruptures include: (1) The formation of corpus luteum cyst. Under normal circumstances, the corpus luteum does not exceed $2 \mathrm{~cm}$, non-cystic, and the formation of corpus luteum abscess usually exceeds $3 \mathrm{~cm}$. The formation of corpus luteum cyst is the main factor for rupture of 
the corpus luteum; (2) External force, gynecological examination, squeezing or sexual intercourse can also cause the rupture of the corpus luteum; (3) Abnormal blood coagulation mechanism, such as rupture of the corpus luteum after receiving chronic anticoagulation therapy.

The rupture of the corpus luteum cyst can lead to abdominal and pelvic hemorrhage, clinical manifestations of sudden lower abdominal pain, significant peritoneal irritation, and a sense of swelling in the anus, and even hemorrhage and hemorrhagic shock. It directly threatens the patients' lives, and most of them require emergency surgery. As some patients have no specific clinical manifestations, they may be misdiagnosed as acute appendicitis, torsion of ovarian cyst pedicle, rupture of ectopic pregnancy and other acute abdomen. Therefore, the correct diagnosis before surgery is very important.

Currently, plain abdominal radiography is still a common and basic diagnostic method for patients with acute abdomen, but its diagnostic value in gynecological acute abdomen is limited, and it is only suitable for excluding common acute abdomen such as intestinal obstruction or pneumoperitoneum. Ultrasound examination has the advantages of simple operation, strong intuitiveness, no radiation and easy repeatability. It has high diagnostic accuracy among common gynecological diseases and has been used for the diagnosis of gynecological acute abdomen. However, as some patients see it as the onset of acute abdomen, when handled by other doctors outside the obstetrics and gynecology department with insufficient knowledge of the disease, it will inevitably lead to missed diagnosis or misdiagnosis. CT examination is often used to differentiate and diagnose acute abdomen. Familiarizing the CT manifestations of ruptured and bleeding corpus luteum cyst is of great significance to help qualitative diagnosis.

CT examination is becoming more and more popular in clinical applications. According to previous reports in the literature, the diagnosis can be achieved by identifying unilateral cystic watery lesions in the appendage area during the period of simple corpus luteum cyst on CT scan, and the CT images show clear edges and mixed density shadows. The cyst wall is thicker, showing "serrated" pattern. Further pathological examinations indicated that the cyst wall is mainly composed of granular cells, and blood vessels are often formed about 3 days after ovulation, and enhanced CT examination shows that the cyst wall is strengthened. Rupture and bleeding of corpus luteum cyst is often accompanied by clot attachment and covering. CT scans can show the appearance of cysts in and around blood clots, and Guo Yiling et al. ${ }^{[3]}$ believed that this $\mathrm{CT}$ sign is characteristic. After the cyst ruptures, intra-abdominal hemorrhage may occur. In previous studies, it has been shown that patients with a bleeding volume of $3000 \mathrm{ml}$ are positively correlated with abundant new blood vessels during the formation of the luteal cyst. Based on the summary of CT imaging of HCLC patients in this paper, it is believed that it has the following characteristics: The edge of the cyst wall is not clear, showing thick-walled enhancement, and mixed density shadows can be seen in the cavity. This is mainly due to the presence of fluid in the abdominal cavity, and the density shadows can be seen in the pelvic cavity, colon, iliac fossa triangle and other areas; There are low-density foci in the appendage area, with discontinuous performance and ruptures, which indicates the presence of active bleeding; The ruptured cyst has a high-density shadow, which is related to blood clot sedimentation after bleeding.

According to the ultrasound signs, the amount of bleeding, and the overall clinical and surgical conditions, it can be divided into three types: type I - superficial type, patients of this type can be treated conservatively, and most do not need surgery; type II - surrounding type, which can adopt elective surgical treatment; Type III heterogeneous mass type, requiring timely surgical treatment. In the course of the disease, the bleeding of the corpus luteum cyst can be classified as: fresh bleeding stage, clot formation stage and hemolysis stage. Ultrasonography can diagnose fresh bleeding and hemolysis in combination with anechoic signs or hypoechoic signs, and can diagnose on clots according to strong echo. Further observing the CT image, combined with the actual clinical situation of the patient, if the CT manifestations were observed to be low-density shadows and the patient has a shorter onset time accompanied by contrast agent spillover, it can be defined as fresh bleeding; if the onset time is longer, perform an enhanced scan to rule out the spillover of the contrast agent, then there is a risk of hemolysis after old bleeding; if CT shows a high- 
density shadow, it suggests the presence of a clot.

The CT features of the 24 patients in this group were analyzed: liquid low-density shadow in the ventricle detected on the ruptured cyst wall, and some of the cysts were accompanied by signs of increased density, indicating the presence of blood clots; ruptured cysts were mainly manifested by low-density lesions, analysis implies that it may be the cause of the fresh bleeding manifestations, the surgery showed the formation of clots around the cyst.

Throughout this paper, although enhanced CT scans have good effects in diagnosing ovaries and the diagnostic accuracy is higher than $80 \%$, which is basically consistent with the study of Shen Yazhi et al ${ }^{[4]}$, it is still worth noting to differentiate from the diagnosis of ectopic pregnancy rupture and bleeding. Ectopic pregnancy is a common occurrence in the ampulla of the fallopian tube. The main clinical history is the history of short menopauses and positive urine HCG. The CT manifestations are mixed-density soft tissue masses in the adnexal area, the edges are not very clear, and the enhanced scan is also uneven, and there are low-density gestational sacs without enhancement, which provides a reference for clinical diagnosis. Moreover, most patients with ruptured corpus luteum cyst do not have vaginal bleeding, while most patients with ectopic pregnancy have vaginal bleeding. Although clinical research is still underway and there is no definite conclusion on the distinction between the two, but in any case, whether it is hemorrhage corpus luteum cyst or ectopic pregnancy, enhanced CT scan can show the pelvic cavity and the situation of abdominal effusion, make a rough assessment on the amount of effusion, and clearly show the spillover of the contrast agent. This is an important reference for determining whether there is active bleeding, clarifying the clinical diagnosis, and guiding the clinical development of scientific and reasonable treatment plans.

In conclusion, the application of enhanced CT scanning in patients with hemorrhage corpus luteum cyst has important value, can provide accurate data reference for clinical treatment, and is worthy of promoting its clinical application.

\section{References}

[1] Liu QH, Liu XZ. An Analysis about Findings and Diagnostic Value of Ultrasonography and CT in Hemorrhage Corpus Luteum Cyst [J]. Chinese Journal of CT and MRI, 2016, 14(11): 92-94.

[2] Liu XY, Lin YN. Clinical analysis of 60 cases of rupture and bleeding of ovarian corpus luteum cyst [J]. Shenzhen Journal of Integrated Traditional Chinese and Western Medicine, 2017, 5(23): 25-26.

[3] Guo YL. Analysis of clinical diagnosis and treatment of 32 cases of ruptured corpus luteum [J]. Shanxi Medical Journal, 2016, 45(12): 1457-1458.

[4] Shen YZ, Fang X, Zhu SQ, et al. CT characteristics of ovarian corpus luteum: a further understanding [J]. Journal of Medical Imaging, 2016, 26(2): 306-309. 